\title{
Medial Orbitofrontal Artery
}

National Cancer Institute

\section{Source}

National Cancer Institute. Medial Orbitofrontal Artery. NCI Thesaurus. Code C33067.

An artery branching from the anterior cerebral artery that supplies the medial portions of the frontal and superior parietal lobes. 